\title{
Four tips to create better figures for scientific papers
}

\section{By Duanduan Han}

$\mathrm{W}$

hen I was a PhD student in chemical engineering, I took for granted the figures I saw and easily understood in papers, until it was my turn to write about my own research results. When I created the first schematic for my experimental procedure, I realized that effective figures require as much effort as the paper itself. Hours passed as I drafted and revised one figure, and then the process started over the next day when I had a better idea. Here are four lessons I have learned about creating figures while earning my $\mathrm{PhD}$.

\section{Learn the fundamentals.}

Scientific figures are judged primarily for their scientific value, but basic artistic concepts can serve as guidelines to create figures that improve science communication. I learned the basics of color theory (e.g., color wheel and color harmony) from art blogs. For example, in a figure or a chart that uses only two colors, choose a pair of complementary colors (e.g., blue and orange, red and green) that are naturally pleasing to the viewer's eyes. Also free online tutorials cover the concept of different perspectives (e.g., one-point

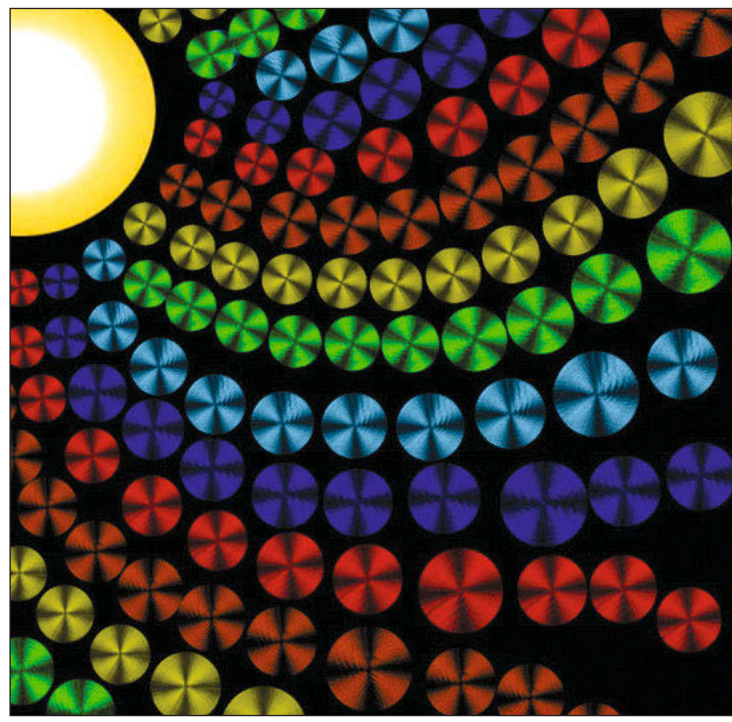

Pseudo-color-processed image of 3D-printed poly(lactic acid) discs observed under crossed polarizers. Credit: Duanduan Han. perspective, two-point perspective), which has helped me with my technical drawings. Increasing your knowledge base on these and other similar areas can help you to plot your ideas quickly and accurately.

\section{Work to find the most suitable illustration software.}

Adobe Illustrator is a powerful piece of software, with specific tools designed to achieve desired effects, such as changing transparency or color gradients. However, it is not the silver bullet that suits the needs of every researcher. I have experience in building three-dimensional (3D) objects as part of my research, and so tend to think in three dimensions when considering scientific figures, which doesn't suit Adobe Illustrator. I eventually discovered the animation software Blender, which is designed to help users create in three dimensions with a wide choice of color schemes and lots of customization options. I use Blender to create 3D models, and then export screenshots of the appropriate perspective as two-dimensional figures for my paper. It helps that Blender is an opensource, free software. When choosing software, try to pick a popular product that is widely used, increasing your chances for assistance if needed.

\section{Get help from others.}

There are plenty of resources available: free training programs at universities or public libraries, short tutorial videos published by software developers and enthusiasts, and online forums where fellow users are willing to answer questions. However, after exploring all of these resources, I still faced frustrating moments. This is the time to search for a mentor, whether a colleague or an online tutor who offers remote services via video conferences. There is no need to struggle alone if resources are available.

\section{Join an art-in-science event.}

Many universities and professional associations organize competitions and exhibitions to showcase artwork created by the scientific community. The Materials Research Society (MRS) has been hosting the Science as Art event as a special feature at MRS meetings since 2014. Visualization methods provide an important tool in science for the analysis and presentation of scientific work. I have seen and enjoyed pseudo-colored microscopic images resembling natural sceneries, everyday objects, and cartoon characters. I have attended and submitted my works to several exhibitions over the years. By visiting such exhibitions, I learned new scientific principles outside of my fields and made new contacts and friends, one of which helped me to learn Blender. Eventually, these insights contributed to a deeper understanding of my research that was reflected in the figures of my papers.

Scientific images can transcend their role as a medium for transmitting information and contain the aesthetic qualities that transform them into objects of beauty and art.

\section{Sources:}

- An art blog about color theory

https://www.colormatters.com/color-and-design/ basic-color-theory

\section{- Science behind complementary colors} https://www.smithsonianmag.com/smart-news/ the-scientific-reason-complementary-colors-lookgood-together-114030051

- An online tutorial about perspective https://www.artistsnetwork.com/art-mediums/ drawing/learn-to-draw-perspective

- Inkscape tutorials https://www.youtube.com/watch?v=8f011wdiW7g \&list=PLqazFFzUAPc5I0QwDoZ4Dw2YSXt07IWNv

- Blender tutorials

https://www.youtube.com/watch?v=TPrnSACiTJ4\& list=PLjEaolNr3zgEqOu2MzVgAaHEBt--xLB6U

\section{- MRS Bulletin Impact article}

"Picturing Science and Engineering," by Felice C Frankel, page 994

Duanduan Han, Texas A\&M University, is a member of the MRS Student Engagement Subcommittee. 